\title{
A database devoted to the insects of the cultural heritage
}

\begin{abstract}
This database, implemented by both the CICRP and the INRA, gathers the most important pests affecting the cultural heritage. These insects represent a serious threat to the preservation of cultural properties such as museum collections, libraries and archives, movable objects and immovable objects in historical buildings.

It is an easy tool for identifying the species of interest.

It also permits very prompt undertaking of the required actions against the infestations. This database is of interest to any professional in charge of the conservation of the cultural heritage along with any other professional or scientist interested in these subjects.
\end{abstract}

Key words: cultural heritage pests, database, molecular identification, infested material.

\section{INTRODUCTION}

The identification of the insects present in cultural conservation sites is essential in order to undertake preventive and curative measures in case of infestation.

Currently, the identification of the insects requires complex keys, different for each taxonomic group, often based on very peculiar morphological characters. This identification becomes even more difficult when considering the different stages of insects. Therefore, identification very often requires the competence of entomologists, specialists of different taxa while actually frequently being a necessity for all kinds of cultural heritage operators.

The Interregional Center for the Conservation and Restoration of the Cultural Heritage of Marseille (CICRP) and the National Institute of Agronomic Research (INRA) of Montpellier, in collaboration with the National Center of DNA sequence (GENOSCOPE) of Evry, have created a database devoted to the "Insects of the Cultural Heritage".

The database is aimed at all professionals in charge of cultural heritage conservation and to any other professional or scientist concerned or interested in these issues.

\section{The contents of the database and headings}

The database inventories and describes the dangerous insects that can represent a 
risk for the conservation of cultural properties such as museum collections, libraries and archives, antique furniture, and historical buildings.

Its contents, its ergonomics and its presentation make the database accessible to any public.

The visual identification and the information on the insects and the damage they cause are easily accessible starting from three headings:

"Insects from A to Z",

"Materials infested", and

"Identification".

For each species several entries are proposed such as the scientific name and synonyms, and the common names in some of the most commonly used languages (Fig. 1), the infested materials (Fig. 1), the keys of identification (dichotomic and visual) (Fig. 2), and the molecular sequence (Fig. 3). In fact, the specificity of this database consists in the possibility of identifying an insect starting from its DNA sequence.
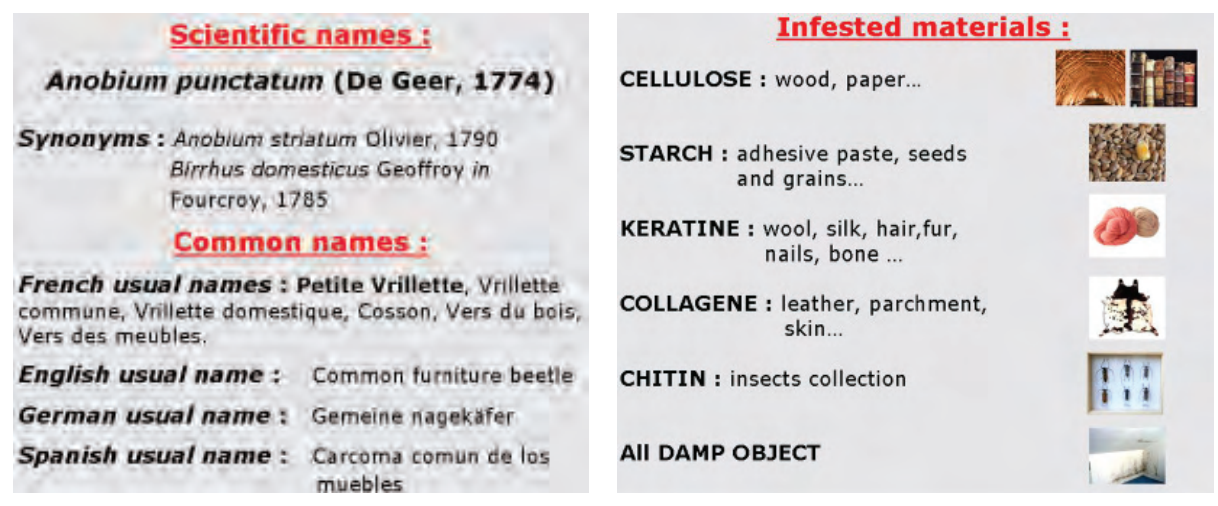

Fig.1 - (left) An example of how to search for an insect (Anobium punctatum) in the database using its common or its scientific name. (right) An example of how to search for an insect in the database from the different substrates it feeds on, e.g. cellulose (wood, paper...) or collagen (leather, skin...).

\section{Identification of the insects by molecular approach}

This entry is dedicated to biologists interested in molecular species identification. This approach is based on the identification of a species from its DNA.

Compared to the morphological identification, the use of the molecular features for taxonomy has many advantages.

First of all, it is not necessary to know morphological terminologies in detail. In addition, the descriptions of the developmental stages of all different insect species are not always available. In some cases, when cryptic species are involved, classical studies 


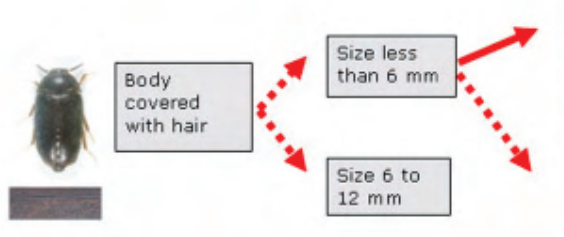

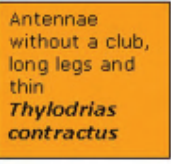

Antennae with club, short legs

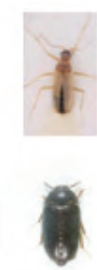

OR
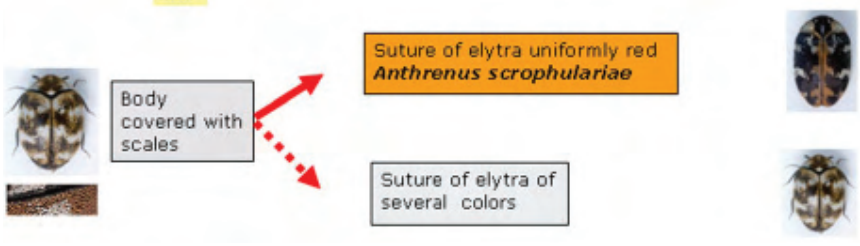

Fig. 2 - An example of an identification key, based on illustrated morphological characters.

\section{Query Sequence (FASTA format):}

AACACTTTATTTCATTTTCCGACCATGATCACGAATACTACCCACTTCATTAAGACTCCTAATTCCACCACAACTACGAAATCC TCCCTCCTTAATTGCACATGACCAAATTTATAATGTTATTGTTACTCCCCACCCATTCATCATAATTTTCTTCATAGTAATACCA ATCCTAATTCCACCATTCCCAAACTCATTACTCCCTTTAATACTACCCCCCCCTCATATACCATTTCCACCAATAAACAACATA AGATTCTGACTACTTNCCCCATCCCTTCACTTCTTCTCATAAGAAGAATTCTTCAAAGACGNCCNCGNACACGGTGAACCGTT TANCCACCCCTCTCATCAAACATTCCACATCGCCGATCCTCTGTTCATCTTCCCATTTTCAGACTACACTTAGCACGAATCTCAT CAATTCTCCCCCCACTTAATTTCATTACAACACTAATCAATATACCACCCCAACCAATAACCCTTCACCCAATACCCCTATTCC TITGACCAGTAGTAATTACTCCAATTTIACTTCTCTTATCTCTTCCTGTITIACCTGGAGCTATTACCATACTCCTTACAGATCG AAACATCAACACATCATTCTTTCACCCTCCTCCCCCACCCCATCCAATCCTTTA

\section{Submit Clear Sequence}

Fig. 3 - An example of a DNA sequence used to search for an insect in the database via a search engine.

based on morphology are difficult or even impossible to use.

Conversely, DNA sequencing allows the identification of all developmental stages of the insects (eggs, larvae, nymphs, adults) as well as the detection of the cryptic species.

Of course this technique will not satisfy all needs of entomologists but will offer them a new tool for identification and treatment of infested material at an early stage of insect invasion.

To achieve this goal we are currently establishing a databank of DNA sequences extracted from specimens accurately identified by entomologists. 
In a few years we think that this technique will allow the identification of the insects in a routine way. Next Generation Sequencing techniques (Illumina, 454 Roche, SOLiD, SMRT) produce millions of sequence reads at increasingly lower prices.

\section{Other documents and information on the insects}

In addition to nomenclature, classification and presentation of orders and families, for each species, a descriptive survey is presented that gives information on its biology, its distribution and frequency, and the possible damage to cultural heritage materials.

The glossary contains the entomological and technical vocabulary used in the database, in order to allow a better comprehension of the texts and descriptions, whatever the level of the person consulting the database, technician or scientist.

Fabien Fohrer, Centre Interrégional de Conservation et de Restauration du Patrimoine (C.I.C.R.P.), 21 rue Guibal 13003 Marseille, France.

E-mail: fabien.fohrer@cicrp.fr

Michel Martinez, Institut National de la Recherche Agronomique (INRA), Centre de Biologie pour la Gestion des Populations (CBGP), Campus international de Baillarguet-CS 3001634988 Montferrier-sur-Lez cedex, Montpellier, France.

E-mail:martinez@supagro.inra.fr

Franck Dorkeld, Institut National de la Recherche Agronomique (INRA), Centre de Biologie pour la Gestion des Populations (CBGP), Campus international de Baillarguet-CS 3001634988 Montferrier-sur-Lez cedex, Montpellier, France.

E-mail: dorkeld@supagro.inra.fr 